\title{
Qualidade de vida dos estudantes da área da saúde que utilizam metodologia ativa de ensino-aprendizagem
}

\author{
Quality of life of healthcare students using active teaching-learning methodology \\ Calidad de vida de estudiantes de salud que utilizan metodología activa de enseñanza-aprendizaje
}

Recebido: 06/04/2021 | Revisado: 15/04/2021 |Aceito: 17/04/2021 | Publicado: 01/05/2021

\author{
Damião da Conceição Araújo \\ ORCID: https://orcid.org/0000-0003-1116-170X \\ Universidade Federal de Sergipe, Brasil \\ E-mail: damiao.araujo92@gmail.com \\ Carla Passos Almeida \\ ORCID: https://orcid.org/0000-0002-6935-8161 \\ Universidade Federal de Sergipe, Brasil \\ E-mail: ccarlapassos@gmail.com \\ Luciana Rodrigues Prata Santana \\ ORCID: https://orcid.org/0000-0002-5074-1208 \\ Universidade Federal de Sergipe, Brasil \\ E-mail: luuh.rps@gmail.com \\ Sabrina Barreto Mota \\ ORCID: https://orcid.org/0000-0003-3508-4964 \\ Universidade Federal de Sergipe, Brasil \\ E-mail: sabrinabarretomb@gmail.com \\ Allan Dantas dos Santos \\ ORCID: https://orcid.org/0000-0002-6529-1887 \\ Name of institution where it operates, Country \\ E-mail: allanufs@hotmail.com \\ Shirley Verônica Melo Almeida Lima \\ ORCID: https://orcid.org/0000-0002-9062-0742 \\ Universidade Federal de Sergipe, Brasil \\ E-mail: shirleymelo.lima@gmail.com \\ Karina Conceição Gomes Machado de Araújo \\ ORCID: https://orcid.org/0000-0003-4433-5227 \\ Universidade Federal de Sergipe, Brasil \\ E-mail: karinaconceicaoaraujo@gmail.com \\ José Antônio Barreto Alves \\ ORCID: https://orcid.org/0000-0002-8647-8050 \\ Universidade Federal de Sergipe, Brasil \\ E-mail: antoniobalves@gmail.com \\ Andreia Centenaro Vaez \\ ORCID: https://orcid.org/0000-0002-1503-0785 \\ Universidade Federal de Sergipe, Brasil \\ E-mail: andreiacentenarovaez@gmail.com
}

\begin{abstract}
Resumo
Objetivo: Avaliar a qualidade de vida dos estudantes de ciências da saúde que utilizam metodologia ativa de ensinoaprendizagem. Metodologia: Estudo observacional do tipo transversal, realizado em uma Universidade Pública situada na região centro-sul do estado de Sergipe. A amostra foi não probabilística por conveniência, constituída por 141 estudantes dos cursos de enfermagem, farmácia, fisioterapia, fonoaudiologia, medicina, nutrição, odontologia e terapia ocupacional. Foi aplicado um instrumento de caracterização e o World Health Organization's Quality of LifeBref. A coleta de dados foi realizada por formulário online enviado para o e-mail de todos os estudantes. Os dados foram armazenados e analisados pelo BioEstat versão 5.0 e GraphPad Prism versão 7.0 Resultados: Os estudantes de ciências da saúde apresentaram escores abaixo da média da qualidade de vida geral e dos domínios físico, psicológico, relações sociais e meio ambiente no primeiro, terceiro e quinto ciclo da graduação. Foi evidenciado que quanto maior o tempo de estudo autodirigido maior a alteração na qualidade de vida, sobretudo, no domínio psicológico. Conclusão: O uso das metodologias ativas altera a qualidade de vida dos estuantes de ciências da saúde em escores variados e dependendo do ciclo da graduação.
\end{abstract}

Palavras-chave: Estudantes de ciências da saúde; Indicadores de qualidade de vida; Aprendizagem baseada em problemas; Ensino; Metodologias ativa de ensino-aprendizagem. 


\begin{abstract}
Objective: To evaluate the quality of life of health science students who use active teaching-learning methodology. Methodology: Observational cross-sectional study, carried out at a Public University located in the south-central region of the state of Sergipe. The sample was non-probabilistic for convenience, consisting of 141 students from the courses of nursing, pharmacy, physiotherapy, speech therapy, medicine, nutrition, dentistry and occupational therapy. A characterization instrument and the World Health Organization's Quality of Life-Bref were applied. Data collection was performed using an online form sent to all students' e-mail. The data were stored and analyzed using BioEstat version 5.0 and GraphPad Prism version 7.0 Results: Health science students had scores below the average of general quality of life and of the physical, psychological, social relations and environment domains in the first, third and fifth cycle of graduation. It was shown that the longer the self-directed study time, the greater the change in quality of life, especially in the psychological domain. Conclusion: The use of active methodologies alters the quality of life of health science students in varied scores and depending on the undergraduate cycle.
\end{abstract}

Keywords: Health sciences students; Quality of life indicators; Problem-based learning; Teaching; Active teachinglearning methodologies.

\title{
Resumen
}

Objetivo: Evaluar la calidad de vida de los estudiantes de ciencias de la salud que utilizan una metodología activa de enseñanza-aprendizaje. Metodología: Estudio observacional transversal, realizado en una Universidad Pública ubicada en la región centro-sur del estado de Sergipe. La muestra fue no probabilística por conveniencia, compuesta por 141 estudiantes de los cursos de enfermería, farmacia, fisioterapia, logopedia, medicina, nutrición, odontología y terapia ocupacional. Se aplicó un instrumento de caracterización y Quality of Life-Bref de la Organización Mundial de la Salud. La recolección de datos se realizó mediante un formulario en línea enviado al correo electrónico de todos los estudiantes. Los datos fueron almacenados y analizados por BioEstat versión 5.0 y GraphPad Prism versión 7.0. Resultados: Los estudiantes de ciencias de la salud obtuvieron puntajes por debajo del promedio de calidad de vida general y de los dominios físico, psicológico, relaciones sociales y medio ambiente en el primer, tercer y quinto ciclo de graduación. Se demostró que cuanto mayor es el tiempo de estudio autodirigido, mayor es el cambio en la calidad de vida, especialmente en el dominio psicológico. Conclusión: El uso de metodologías activas altera la calidad de vida de los estudiantes de ciencias de la salud en puntajes variados y dependiendo del ciclo de pregrado.

Palabras clave: Estudiantes de ciencias de la salud; Indicadores de calidad de vida; Aprendizaje basado en problemas; Ensenãnza; Metodologías activas de enseñanza-aprendizaje.

\section{Introdução}

As demandas da sociedade em relação aos problemas de saúde e oferta de serviços resolutivos, promoveu uma mudança na formação de profissionais da área da saúde. O método de ensino tradicional, focado na transmissão de conhecimentos pelo professor como responsável ativo nas aulas, deixam espaço para o método inovador das metodologias ativas, onde os alunos adquirem habilidades para aprender, construir o seu conhecimento e desenvolver habilidades importantes para a prática profissional como a comunicação, raciocínio clínico e trabalho em equipe (Souza et al., 2020).

As metodologias ativas cresceram no espaço das universidades e proporciona aos alunos o desenvolvimento de habilidades-técnicas e competências, com a finalidade de ofertar uma formação acadêmica mais desafiadora (Abate et al., 2003). Exemplos de métodos de aprendizagem ativa incluem avaliação de estudos de caso, discussões em classe, aprendizagem baseada em projetos, aprendizagem baseada em problemas, simulação (interpretação de papéis, paciente simulado e paciente virtual), aprendizagem baseada em jogos e construção de mapas conceituais (Medina et al., 2013).

A metodologia ativa estimula constantemente a aprendizagem do estudante, uma vez que utiliza a resolução de problemas através de casos clínicos prévios, que retratam o cenário clínico e social que o aluno estará inserido após a sua formação. A autonomia é desenvolvida para que o aluno pesquise o conteúdo em fontes bibliográficas de alto nível, estude os conteúdos, questione a realidade social e de saúde dos diversos grupos, elabore estratégias de solução para determinado problema de um indivíduo, família e comunidade, bem como trabalhe de forma colaborativa com outras áreas (Colares et al., 2018).

O ingresso do estudante na vida acadêmica traz consigo mudanças sociais e de saúde significativas que podem estar associados ao modelo de ensino-aprendizagem adotado pela instituição (Caballero et al., 2006), promovendo a necessidade de adaptação do aluno a nova rotina universitária e o desenvolvimento da capacidade de lidar com as emoções, a autonomia, 
relações interpessoais e interdependência (Cunha et al., 2005; Bento et al., 2007; Cabral et al., 2010; Teixeira et al., 2008). No entanto, o aluno que utiliza método ativo de ensino-aprendizagem pode apresentar sintomas negativos durante o processo. As atividades e créditos exigidos, quantidade de conteúdo para estudo dirigido, participação em cenários de vivência do futuro profissional desencadeia a sobrecarga acadêmica, estresse, e sentimento de incapacidade (Rocha et al., 2019).

Desta forma, a qualidade de vida (QV) dos estudantes sofre modificações ao longo do processo de graduação nos aspectos físico, psicológico, relações sociais e condições ambientais, principalmente, quando inicia o contato com a prática profissional em níveis de complexidade variados. As cobranças por prazos e demandas maiores para estudo podem diminuir o tempo para a convivência social e práticas de lazer (Rocha et al., 2019). Os professores que atuam no contexto do método ativo como facilitador e tutor, bem como a instituição de ensino, precisam observar a condição de qualidade de vida dos estudantes para promover ações que reduzam os sentimentos negativos (Souza et al., 2018).

Destarte, o objetivo do estudo foi avaliar a qualidade de vida dos estudantes de ciências da saúde que utilizam metodologia ativa de ensino-aprendizagem.

\section{Metodologia}

\subsection{Delineamento e aspectos éticos do estudo}

Estudo observacional do tipo transversal, realizado em uma Universidade Pública situada na região centro-sul do estado de Sergipe, aprovado pelo Comitê de Ética e Pesquisa da Universidade Federal de Sergipe sob o protocolo de número CAAE: 61993616.3.0000.5546. O campus de Ciências da Saúde Professor Antônio Garcia Filho, da Universidade Federal de Sergipe, oferece oito cursos: enfermagem, farmácia, fisioterapia, fonoaudiologia, medicina, nutrição, odontologia e terapia ocupacional. O ingresso dos alunos é anual. O campus utiliza em todos os cursos as metodologias ativas de ensinoaprendizagem, com destaque para a Aprendizagem Baseada em Problemas (ABP), que estruturam o currículo em módulos, disciplinas e subunidades curriculares. O sistema funciona por ciclos anuais ( 1 ao 5); ressalta-se que no $1^{\circ}$ ciclo as aulas ocorrem com a junção de todos os cursos, promovendo o contato e conhecimento profissional das diversas áreas. Após esse período cada curso conduz o direcionamento específico em virtude das demandas pedagógicas e de formação. As aulas são estruturadas com dez alunos e um professor tutor para a discussão em grupo dos conteúdos. Desde o primeiro ano do curso os estudantes têm contato com problemas baseados na realidade profissional, bem como são introduzidos na comunidade para acompanhar os serviços de saúde e ofertar ações que resolvam os problemas identificados.

\subsection{Amostra e critérios de elegibilidade}

A população foi composta por estudantes dos oito cursos de ciências da saúde ofertado pela instituição de ensino: enfermagem, farmácia, fisioterapia, fonoaudiologia, medicina, nutrição, odontologia e terapia ocupacional. A amostra foi não probabilística por conveniência, que após cálculo foi constituída por 141 estudantes.

Os seguintes critérios foram utilizados para o recrutamento e inclusão na pesquisa: (1) aluno matriculado em um dos cursos de ciências da saúde ofertados pela instituição pública de ensino: enfermagem, farmácia, fisioterapia, fonoaudiologia, medicina, nutrição, odontologia e terapia ocupacional; (2) cursando um dos ciclos/semestres: I, II, III, IV ou V ano; (3) frequentando as aulas sem dispensa de nenhum componente curricular; e (4) questionários adequadamente respondidos.

\subsection{Variáveis e instrumentos}

As variáveis investigadas e os desfechos de interesse para o estudo foram: (1) dependentes (desfecho primário): qualidade de vida dos estudantes; (2) independentes (co-variáveis exploratórias): gênero, raça/cor da pele, estado civil, curso, ciclo/semestre, bolsa auxílio da universidade, hora de estudo autodirigido e os domínios do WHOQOL-Bref: físico, 
psicológico, relações sociais e meio ambiente.

Os instrumentos utilizados foram um questionário sobre o perfil sociodemográfico dos estudantes elaborado pelos pesquisadores; e do World Health Organization's Quality of Life- Bref (WHOQOL-bref). O WHOQOL-bref é um instrumento objetivo, de fácil aplicação e recomendado pela Organização Mundial da Saúde (OMS). Foi adaptado, aplicado e validado para o Brasil e inclui quatro domínios relacionados à QV: físico, psicológico, relações sociais e meio ambiente (Fleck et al., 2000).

\subsection{Sistemática de coleta de dados}

A coleta de dados foi realizada no período de janeiro e fevereiro de 2016 por meio da aplicação de um questionário on-line sobre o perfil sociodemográfico dos estudantes e do World Health Organization's Quality of Life- Bref (WHOQOLbref). Os instrumentos foram enviados para todos os alunos por e-mail através da coordenação geral da universidade, no formato de um formulário on-line, juntamente com o termo de consentimento livre e esclarecido (TCLE); e informações sobre a pesquisa.

Para evitar potenciais fontes de viés, o formulário foi configurado para que fosse preenchido uma única vez pelo voluntário, alertasse sobre a ausência de alguma alternativa não preenchida e programado para evitar duplicidade de envio. O instrumento foi enviado em dois momentos distintos para aumentar a probabilidade de adesão dos acadêmicos na pesquisa, sendo retirado da lista os e-mails dos alunos que já haviam respondido o questionário. A tabulação de dados foi realizada por dois pesquisadores treinados e confrontados para identificar inconsistências.

\subsection{Análise estatística}

Os dados foram armazenados e analisados pelo programa BioEstat versão 5.0 e apresentados sob a forma de tabelas e gráficos. Estes foram elaborados com o auxílio do programa GraphPad Prism versão 7.0. Inicialmente, realizou-se a estatística descritiva dos dados. As variáveis numéricas foram calculadas por meio das medidas de tendência central e de dispersão. As variáveis categóricas foram apresentadas em frequência absoluta e relativa. O escore do WHOQOL-bref foi calculado usando a sintaxe recomendada pela OMS em uma escala de 0 a 100, sendo a maior pontuação indicativa de melhor QV (Fleck et al., 2000).

Foi utilizado o teste do Qui-quadrado de Pearson para a associação entre as variáveis categóricas. Os escores foram divididos em cinco grupos para aplicar o teste não-paramétrico de Mann Whitney cuja finalidade foi comparar a QV em cada ciclo/semestre. O teste de regressão linear simples foi aplicado para verificar a influência do tempo de estudo autodirigido com os domínios físico, psicológico, das relações sociais e do meio ambiente da QV dos estudantes. A regressão linear múltipla foi utilizada para verificar o quanto a pontuação de cada domínio do WHOQOL-Bref influencia na qualidade de vida global. Assim foi apresentado o coeficiente de regressão e o coeficiente de determinação ajustado $\left(R^{2}\right)$. Para todo o estudo, foi considerado o valor de $\mathrm{p}<0.05$ de significância.

\section{Resultados}

O perfil sociodemográfico dos estudantes revelou que a maior parte eram do gênero feminino $(78.72 \%)$, que se autodeclararam pardos (58.16\%), solteiros (89.36\%), cursando enfermagem (25.53\%), no primeiro ciclo (24.82\%) e que não recebem bolsa auxílio da universidade $(61.70 \%)$. 
Research, Society and Development, v. 10, n. 5, e15410514737, 2021

(CC BY 4.0) | ISSN 2525-3409 | DOI: http://dx.doi.org/10.33448/rsd-v10i5.14737

Tabela 1. Distribuição do perfil sociodemográfico dos estudantes de ciências da saúde.

\begin{tabular}{|c|c|c|c|c|}
\hline Varáveis & Categorias & $\mathbf{n}$ & $\%$ & $\mathbf{p} \dagger$ \\
\hline \multirow[t]{2}{*}{ Gênero } & Masculino & 30 & 21.28 & 0.00 \\
\hline & Feminino & 111 & 78.72 & \\
\hline \multirow[t]{4}{*}{ Raça/cor da pele } & Branca & 38 & 26.95 & 0.00 \\
\hline & Preta & 19 & 13.48 & \\
\hline & Parda & 82 & 58.16 & \\
\hline & Amarela & 2 & 1.42 & \\
\hline \multirow[t]{3}{*}{ Estado civil } & Nunca se casou & 126 & 89.36 & 0.00 \\
\hline & Casado & 14 & 9.93 & \\
\hline & Separado/divorciado & 1 & 0.71 & \\
\hline \multirow[t]{8}{*}{ Curso } & Enfermagem & 36 & 25.53 & 0.02 \\
\hline & Medicina & 15 & 10.64 & \\
\hline & Fisioterapia & 23 & 16.31 & \\
\hline & Farmácia & 20 & 14.18 & \\
\hline & Terapia Ocupacional & 16 & 11.35 & \\
\hline & Odontologia & 13 & 9.22 & \\
\hline & Nutrição & 14 & 9.93 & \\
\hline & Fonoaudiologia & 4 & 2.84 & \\
\hline \multirow[t]{5}{*}{ Ciclo } & Primeiro & 35 & 24.82 & 0.01 \\
\hline & Segundo & 31 & 21.99 & \\
\hline & Terceiro & 30 & 21.28 & \\
\hline & Quarto & 23 & 16.31 & \\
\hline & Quinto & 22 & 15.60 & \\
\hline \multirow[t]{2}{*}{ Bolsa remunerada } & Sim & 54 & 38.30 & 0.00 \\
\hline & Não & 87 & 61.70 & \\
\hline Total & & 141 & 100 & \\
\hline
\end{tabular}

$\dagger$ Teste do Qui-quadrado de Pearson $(\mathrm{p}<0.05)$. Fonte: Autores.

Os escores do WHOQOL-Bref variou em relação ao ciclo dos estudantes. A QV geral e domínios físico, psicológico, relações sociais e meio ambiente apresentaram pontuações menores no primeiro, terceiro e quinto ciclo indicando alteração nos aspectos da QV com escores menores que a média de 50 pontos da amostra (Figura 1). 
Figura 1. Comparação da média dos escores da qualidade de vida geral e dos domínios físico, psicológico, das relações sociais e do meio ambiente do WHOQOL-Bref, em relação ao ciclo/semestre dos estudantes.

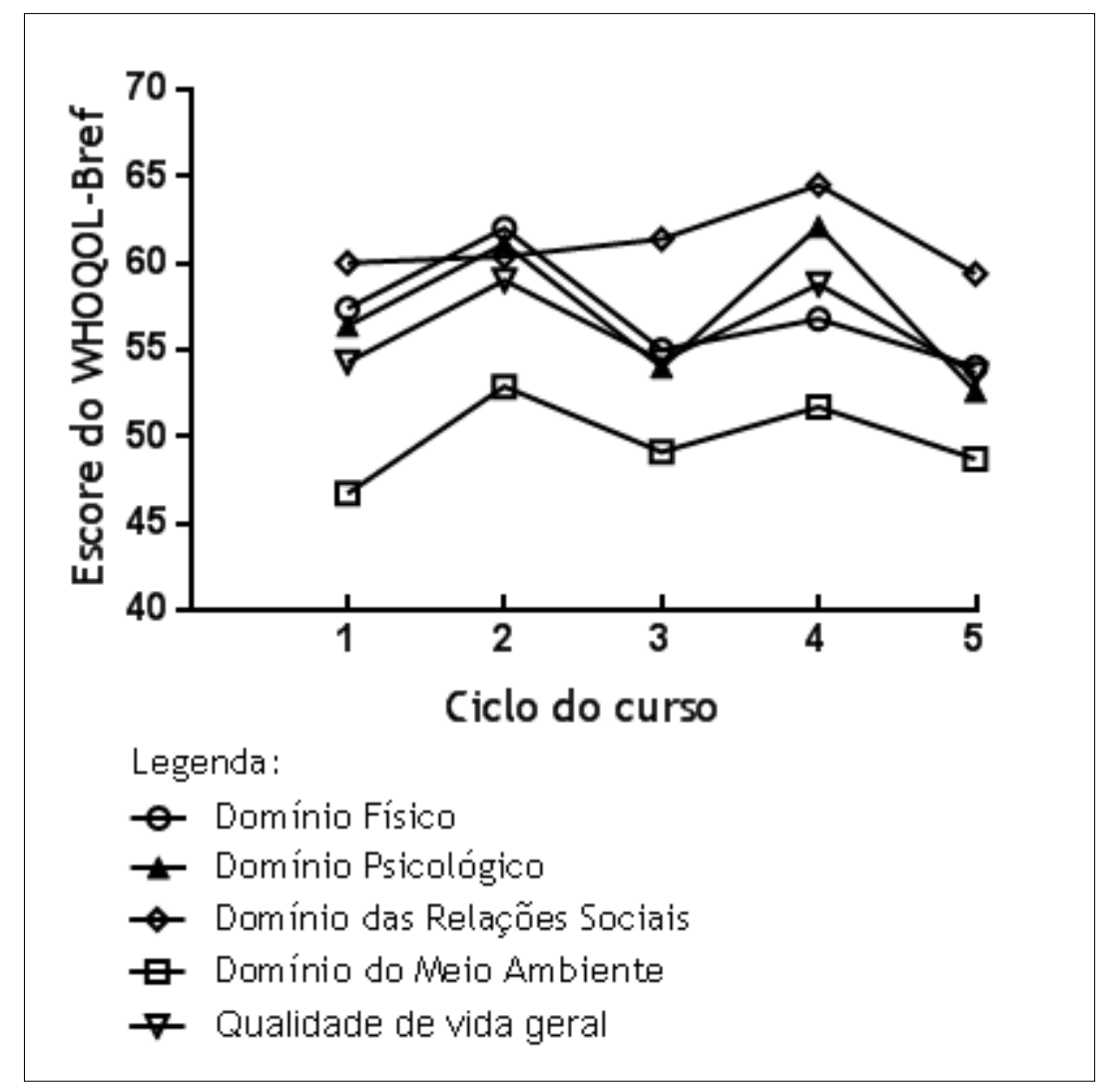

Teste não-paramétrico de Mann-Whitney ( $\mathrm{p}<0.05)$. Fonte: Autores.

Na Figura 2 observa-se a relação dos escores dos domínios do WHOQOL-Bref com o tempo de estudo autodirigido de duas (2), quatro (4), doze (12) e quinze (15) horas informado pelos estudantes. Verificou-se diminuição da pontuação dos escores nos domínios da QV dos estudantes que possuem maior tempo no estudo autodirigido. O domínio psicológico apresenta maior alteração em relação aos valores (média: 40.0). 
Figura 2. Regressão da média dos domínios do WHOQOL-Bref em relação ao tempo de estudo autodirigido dos estudantes. Teste de regressão linear simples $(\mathrm{p}<0.02)$.

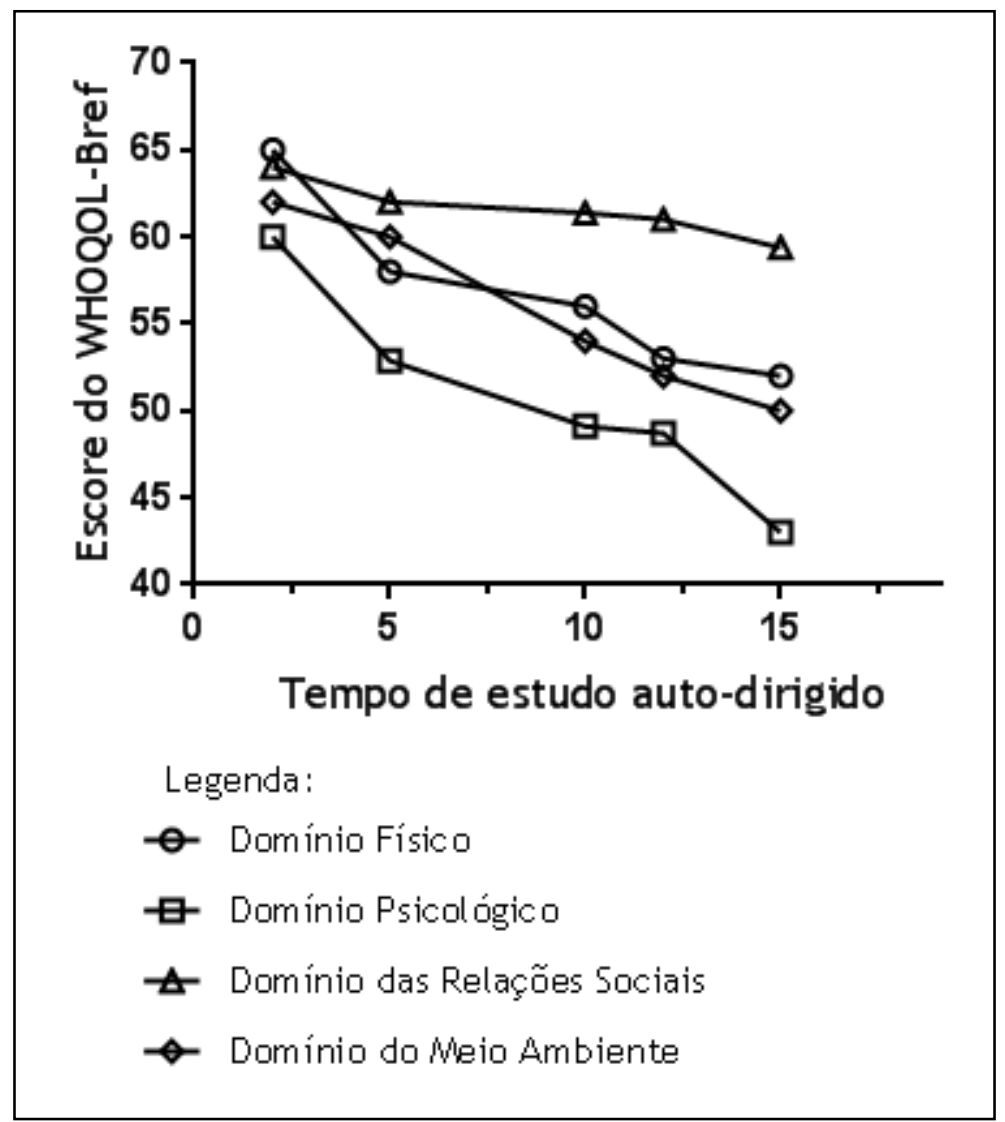

Regressão linear simples ( $\mathrm{p}<0.05)$. Fonte: Autores.

A análise do WHOQOL-Bref com o uso da regressão linear múltipla, permitiu verificar o quanto cada valor dos domínios influenciou na alteração da QV global dos estudantes por ciclo. Foi evidenciado nos coeficientes de determinação $\left(\mathrm{R}^{2}\right)$ o valor preditor forte em todos os domínios. Em razão disso, verificou-se uma alteração global na QV geral dos estudantes. Além disso, foi identificado maior média de domínio físico em alunos do primeiro ciclo, enquanto o domínio das relações sociais apresenta a menor média. O domínio psicológico apresentou maiores valores entre os alunos do quarto ano e o domínio do meio ambiente oscila a cada ano/semestre analisado com médias maiores no segundo e quarto ciclo (Tabela 2). 
Tabela 2. Distribuição da média, desvio-padrão e valores da regressão linear múltipla dos escores por domínio do WHOQOLBref dos estudantes

\begin{tabular}{|c|c|c|c|c|}
\hline Varáveis & Média/Dp & $\mathbf{R}^{*}$ & $\mathbf{R}^{2 *}$ & $\overline{p \dagger}$ \\
\hline \multicolumn{5}{|l|}{ Primeiro ciclo } \\
\hline Domínio físico & $57.4 \pm 19.4$ & 0.9282 & 86.16 & 0.00 \\
\hline Domínio psicológico & $56.4 \pm 18.4$ & 0.8850 & 78.32 & 0.00 \\
\hline Domínio das relações sociais & $56.9 \pm 25.9$ & 0.9867 & 97.36 & 0.00 \\
\hline Domínio do meio ambiente & $46.7 \pm 16.8$ & 1.0000 & 100.00 & 0.00 \\
\hline \multicolumn{5}{|l|}{ Segundo ciclo } \\
\hline Domínio físico & $61.6 \pm 17.8$ & 0.9277 & 86.07 & 0.00 \\
\hline Domínio psicológico & $61.1 \pm 20.3$ & 0.9548 & 91.16 & 0.00 \\
\hline Domínio das relações sociais & $60.4 \pm 20.5$ & 1.0000 & 100.00 & 0.00 \\
\hline Domínio do meio ambiente & $52.9 \pm 17.3$ & 0.9718 & 94.44 & 0.00 \\
\hline \multicolumn{5}{|l|}{ Terceiro ciclo } \\
\hline Domínio físico & $55.0 \pm 18.4$ & 0.9762 & 95.29 & 0.00 \\
\hline Domínio psicológico & $54.0 \pm 20.2$ & 0.9350 & 87.42 & 0.00 \\
\hline Domínio das relações sociais & $61.4 \pm 22.0$ & 0.9981 & 99.63 & 0.00 \\
\hline Domínio do meio ambiente & $49.1 \pm 19.1$ & 0.9548 & 91.16 & 0.00 \\
\hline \multicolumn{5}{|l|}{ Quarto ciclo } \\
\hline Domínio físico & $56.8 \pm 17.1$ & 0.9137 & 83.48 & 0.00 \\
\hline Domínio psicológico & $62.1 \pm 17.8$ & 0.8637 & 74.60 & 0.00 \\
\hline Domínio das relações sociais & $64.5 \pm 16.5$ & 1.0000 & 100.00 & 0.00 \\
\hline Domínio do meio ambiente & $51.7 \pm 16.1$ & 0.9522 & 90.67 & 0.00 \\
\hline \multicolumn{5}{|l|}{ Quinto ciclo } \\
\hline Domínio físico & $53.7 \pm 15.4$ & 0.9900 & 98.00 & 0.00 \\
\hline Domínio psicológico & $52.6 \pm 14.3$ & 0.9681 & 93.72 & 0.00 \\
\hline Domínio das relações sociais & $59.4 \pm 21.7$ & 0.8916 & 79.49 & 0.00 \\
\hline Domínio do meio ambiente & $48.7 \pm 10.2$ & 1.0000 & 100.00 & 0.00 \\
\hline
\end{tabular}

*Teste de Regressão linear múltipla (p <0.05) com intervalo de confiança (IC) de 95\%; Coeficiente de regressão (R); Coeficiente de determinação ajustado $\left(\mathrm{R}^{2}\right)$; †Teste de Qui-quadrado de Pearson $(\mathrm{p}<0.05)$. Fonte: Autores.

\section{Discussão}

Os resultados deste estudo foram coletados após a implementação do campus de Ciências da Saúde Professor Antônio Garcia Filho da Universidade Federal de Sergipe. É o único campus da universidade que utiliza as metodologias ativas de ensino-aprendizagem sendo promissor na formação dos futuros profissionais. Desta forma, foi importante investigar o impacto das metodologias ativas na QV dos estudantes, sobretudo, em quais domínios ocorrem as alterações, para servir de linha de base no delineamento de ações e aprimoramento das estratégias de enfrentamento.

As metodologias ativas em saúde usufruem de potencialidades inovadoras, nas quais os estudantes desenvolvem uma postura crítica e reflexiva sobre a realidade profissional demonstrada, mantendo uma conduta ativa no processo de aprendizagem. Além disso, as metodologias devem estimular no discente a curiosidade, a criatividade e a problematização, tornando-os aptos à resolução de impasses na prática. O estudante adulto-jovem apresenta receptividade às novas tecnologias e mudanças no cenário de ensino e aprendizagem, pressupondo que a estratégia da metodologia ativa é adequada. Os modelos 
tradicionais que colocam o professor como protagonista do processo de ensinar e aprender tornam-se pouco estimulantes na busca de novos conhecimentos, deixando de atender as expectativas dos alunos e do mundo globalizado (Colares et al., 2018).

A QV envolve questões que podem alterar a percepção do sujeito, os sentimentos e os comportamentos, bem como a condição de saúde. O instrumento mais utilizado para mensurar a QV dos indivíduos, World Health Organization Questionnaire for Quality of Life-Brief Form (WHOQOL-bref), que foi elaborado pela OMS, avalia a QV conforme os seguintes domínios: físico, psicológico, relações sociais e meio ambiente (WHO, 2013).

Neste estudo, foi evidenciado alteração na QV geral e nos domínios físico, psicológico, relações sociais e meio ambiente no primeiro, terceiro e quinto ciclo. A QV dos acadêmicos pode variar de acordo com o ciclo em virtude das atividades exigidas (Santos et al.,2017; Freitas et al., 2018). No campus investigado, os alunos do primeiro ciclo passam por mudanças importantes que alteram a rotina anterior devido a mudança de cidade ou deslocamento diário entre municípios vizinhos para cursar o ensino superior. O contato inicial com a metodologia ativa provoca medo e insegurança na maioria dos discentes, além de estar num ambiente novo e desconhecido. As alterações nos domínios no terceiro ano são decorrentes de um novo processo acadêmico: o ano das especialidades, onde os alunos têm contato maior com os pacientes, por meio do estágio, aumentando sua responsabilidade quanto ao futuro profissional e a demanda de estudos. As alterações no último ano são provocadas pelo término do próprio curso, a conciliação do estágio supervisionado com a construção do trabalho de conclusão de curso e insegurança sobre o mercado de trabalho. O domínio psicológico alterado representa os sentimentos negativos experimentados durante o processo de graduação imposto. Além disso, a maior parte dos estudantes não praticam atividade de lazer e se afastam do convívio social devido as demandas acadêmicas alterando o domínio das relações sociais.

O uso do estudo autodirigido é ferramenta primordial para os alunos do método ativo e está associado à prontidão para a autodireção da aprendizagem, à autoestima e à autonomia do estudante. A eficácia da aprendizagem autodirigida aponta que os estudantes no processo ativo buscam informações em diferentes fontes de dados, compararam a informações coletadas e constroem uma constantemente o raciocínio clínico e crítico para a realidade social e profissional (Oliveira et al., 2009).

Neste estudo, observou-se que quando a variável tempo de estudo autodirigido aumenta, os domínios diminuem. Entretanto, o domínio psicológico destaca-se com maior declínio, sugerindo que os estudantes universitários são vulneráveis ao sofrimento psíquico e desenvolvimento de doenças mentais devido à inúmeros fatores, dentre eles, a autocobrança em obter resultados e ser reconhecido, medo e cansaço. Estudo identificou que 79,8\% de universitários relataram passar por dificuldades emocionais, sendo a ansiedade a mais prevalente (58,36\%) (Castro et al., 2017).

Dados da OMS demonstram que aproximadamente 9,3\% dos brasileiros possuem transtorno de ansiedade, sendo a maior parte jovens universitários. A mudança de rotina e exigência do curso superior somado a experiência pedagógica da metodologia ativa pode influenciar no aparecimento da ansiedade (Touso et al., 2020). O estresse é um dos sentimentos negativos mais frequente em universitários podendo evoluir para a Síndrome de Burnout, resultando em uma diminuição da capacidade dos estudantes se adaptar no método de estudo além da exaustão emocional. Os fatores estressores no meio acadêmico mais comuns são as quantidades de disciplinas e rotina de estudo, competitividade, relacionamento interpessoal, contato com a realidade profissional desde os primeiros anos da graduação, afastamento das atividades sociais e ausência de atividade física (Touso et al., 2020; Cazolari et al., 2020).

O estudo apresenta algumas limitações: a amostra calculada foi projetada para os alunos do campus universitário, não apresentando resultados para cada curso. Assim, sugerimos a necessidade de estudos que investiguem a QV e os fatores associados para cada curso do campus para compreender a demanda e impacto de cada área da saúde no processo de graduação. Contudo, os resultados apresentados podem orientar medidas de atendimento aos alunos para minimizar os sentimentos negativos e alteração na QV. É importante que a instituição e professores forneçam apoio e orientações adequadas para melhorar a QV e, consequentemente, prevenção de estresse, ansiedade, depressão e Burnout. 


\section{Conclusão}

O uso de metodologia ativa de ensino-aprendizagem influenciou na QV dos acadêmicos dos cursos de ciências da saúde, sobretudo, nos domínios psicológico e das relações sociais. Além disso, o tempo em horas dedicado no estudo autodirigido exigido no método também altera a QV.

\section{Referências}

Abate, M. A., Stamatakis M. K., \& Haggett R. R. (2003). Excellence in curriculum development and assessment. Am J Pharmac Educ, 67(3).

Bento, A., \& Mendes, G. (2007). A transição do ensino secundário para o ensino superior: factores contributivos para uma boa adaptação e relação com o sucesso académico universitário [Internet]. In: Anais do IX Congresso da Sociedade Portuguesa de Ciências da Educação: 2007, Legis Editora, 245-51

Caballero, C., Llanos, R. A., \& Sañudo, J. P. (2006). Burnout, engagement y rendimiento académico entre estudiantes universitarios que trabajan y aquellos que no trabajan. Revista Psicogente, 9(16):11-27.

Cabral, J., \& Matos, P. M. (2010). Preditores da adaptação à universidade: o papel da vinculação, desenvolvimento psicossocial e coping. Psychologica, 52(1):55-77.

Castro, A. R. (2017). Reflexões sobre a saúde mental do estudante universitário: estudo empírico com estudantes de uma instituição pública de ensino superior. Revista Gestão em Foco-Edição no 9.

Cazolari, P. G., Cavalvante, M. S., Demarzo, M. M. P., Cohrs, F. M., Sanudo, A. \& Schveitzer, M. C. (2020). Níveis de Burnout e Bem-Estar de Estudantes de Medicina: um Estudo Transversal. Rev. bras. educ. med, 44(4).

Chagas, N. B., Sanches, F. B., Silva, R. F., Melo, D. G., Germano, C. M. R. \& Avó, L. R. S. (2018). Qualidade de Vida de Estudantes de Medicina em um Curso que Adota Metodologias Ativas de Ensino-Aprendizagem. Revista brasileira de educação médica, 42 (4): $96-102$.

Colares, K. T. P. \&amp , Oliveira, W. (2018). Metodologias Ativas na formação profissional em saúde: uma revisão. Revista SUSTINERE, 7(2).

Cunha, S. M., \& Carrilho, D. M. (2005). O processo de adaptação ao ensino superior e o rendimento acadêmico. Psicologia Escolar e Educacional, 9(2):21524.

Fleck, M. P. A., Sérgio Louzada, S., Xavier, M., Chachamovich, E., Vieira, G., Santos, L. \& Pinzon, V. (2000). Aplicação da versão em português do instrumento abreviado de avaliação da qualidade de vida "WHOQOL-bref". Rev. Saúde Pública, 34(2):178-183.

Medina, M. S., Plaza, C. M., Stowe, C. D., Robinson, E. T., DeLander, G., Beck, D. E., et al. (2013) Center for the Advancement of Pharmacy Education (CAPE) Educational Outcomes. Am J Pharm Educ, 2(1).

Oliveira, A. L. (2009). A auto-eficácia para a aprendizagem autodirigida como pilar fundamental da educação e aprendizagem ao longo da vida: Continuação dos estudos de validaçãodo Self-Efficacy for Self-Directed Learning Questionnaire. Psycologica, 51(2)57-71.

Rocha, L., Rubim, L. G., Bernardino, F. M. \& Duarte, M. S. Z. (2019). Qualidade de vida e depressão: estudo comparativo entre etapas no curso de medicina em metodologia ativa. Revista Eletrônica Acervo Saúde, 11(11).

Santos, B. O., \& Bittencourt, F. O. (2017). Análise da Qualidade de Vida e fatores associados dos Acadêmicos da área de saúde de uma Faculdade Particular. Id on Line Rev. Palc, 10(33).

Souza, E. F. D., Silva, A. G. \& Silva, A. I. L. F. (2018). Active methodologies for graduation in nursing: focus on the health care of older adults. Ver. Bras Enferm, 71(2):920-4.

Souza, L. S., Santos, D. A. N. \& Murgo, C. S. (2020). Metodologias ativas na educação superior brasileira em saúde. Revista Internacional, 7(1).

Teixeira, M. A. P., Dias, A. C. G., Wottrich, S. H., \& Oliveira, A. M. (2008). Adaptação à universidade em jovens calouros. Psicol. Esc. Educ. (Impr.), 12(1): 185-202.

Touso, M. F. S., Silva, G. A. B., Carvalho, I. N. \& Alves, G. F. (2020). Adaptação do estudante de medicina no primeiro ano da graduação: fatores protetores e estressores e risco da síndrome de burnout. Rev. Nova Paideia - Revista Interdisciplinar em Educação e Pesquisa Brasília/DF, $2(2): 93$ - 112.

Trópia, P. V., Silva, L. B., Mariano, A. S., Mathias Filho, R., Maia, A. S., \& Gomes, D. C. et al. (2018). V Pesquisa Nacional de Perfil Socioeconômico e Cultural dos (as) Graduandos (as) das IFES. Fórum Nacional, 6(2): 300-320.

Venturini, A. C. (2017). A presença das mulheres nas universidades brasileiras: um panorama de desigualdade. Seminário Internacional Fazendo Gênero 11 \& 13thWomen's Worlds Congress (Anais Eletrônicos).

World Health Organization. (2013). Global status report on road safety 2013: supporting a decade of action. Luxembourg: WHO. 